\title{
Comparison of Foot-Over Bridge with Different Configuration of Members

\author{
${ }^{\text {*1} D r . ~ P r a s h a n t ~ D . ~ H i w a s e, ~}{ }^{2}$ S Venkat Shubham, ${ }^{3}$ Ashlesh S Reddy, ${ }^{4}$ Arshad A Ali \\ ${ }^{1,2,3,4}$ Dept. of Civil Engineering, Shri Ramdeobaba College of Engineering and Management Nagpur (M.S.), \\ India \\ Email: prashant.hiwase@gmail.com,vshubham09@gmail.com,ashleshreddy173@gmail.com, \\ arshadalis12s@gmail.com
}

Received: 06th November 2019, Accepted: 10th February 2020, Published: 29th February 2020

\begin{abstract}
Foot over bridge is an important civil engineering structure designed for the free movement of pedestrians in heavy vehicle traffic regions across roads, railway line etc.

There is a critical comparison between foot over bridges having different configurations based on Strength, Safety, Economy and Sustainability using the concept of Influence Line Diagram (ILD). The comparison between compression and tension members having equal forces has been highlighted by designing and cost comparing of both.
\end{abstract}

\section{Keywords}

IS 800-2007, Influence Line Diagram (ILD), Configuration of Trusses, Tension \& Compression

\section{Introduction}

Foot-over bridge is a walkway bridge designed for the free movement of pedestrian across railway line canals and marshy land etc. Foot over bridge are also located across roads for free movements of pedestrians in high Vehicular traffic region. [8]

\section{Methodology}

Foot-over Bridge is a civil engineering structure, consisting of different structural members in which truss members carries the major stresses. [6] For analysing the structure we have selected four different configurations of trusses. A standard load (constant Live Load \& Dead Load) is applied on all the foot-over bridges by keeping all the parameters constant (span, walk-way, height of truss etc) for determining the stresses in the members and hence selecting the most suitable configuration based on safety and sustainability. [3,7]

\section{Problem Statement}

Comparison of different configurations of foot-over bridge as shown in fig A, B, C \& D, by analysing \& designing Bottom Chord, Top Chord, Vertical \& Inclined members using IS 800-2007. The different configurations of truss members are as shown in the figure A, B, C, D

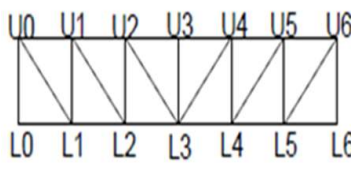

Figure A

Mirrored N-Type Footover Bridge

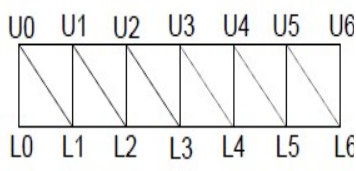

Figure B

N-Type Foot-over Bridge

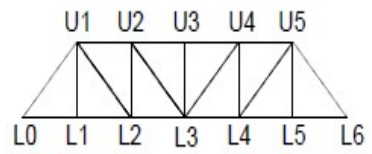

Figure C

Mirrored N-Type

Triangular Foot-over Bridge

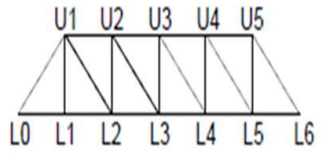

Figure D

N-Type Triangular Foot-over Bridge

Standardised Data $[2,5]$

Span length $=2 \mathrm{~m}$, No of Panels $=6$, Walkway $=2.4 \mathrm{~m}$, Height of Truss $=2 \mathrm{~m}$

Cross girder: Bearing $=0.1 \mathrm{~m}$, Effective length $=2.5 \mathrm{~m}$, Self-weight $=0.1 \mathrm{KN} / \mathrm{m}$, Thickness of flooring $=0.1 \mathrm{~m}$, TRUSS: Self weight $=0.1 \mathrm{KN} / \mathrm{m}$, Live load intensity $=4 \mathrm{KN} / \mathrm{m}^{2}$,

$\mathrm{fu}=410 \mathrm{~N} / \mathrm{mm}^{\wedge} 2$, fy $=250 \mathrm{~N} / \mathrm{mm}^{\wedge} 2$, bolt diameter $=18 \mathrm{~mm}$, pitch $=2.5 \mathrm{~d}=50 \mathrm{~mm}$, edge distance $=1.5 \mathrm{~d} 0=30 \mathrm{~mm}$, gm0=1.1, gmb=1.25: As per IS 800-2007 and Indian steel table 
Table 1: Loading Calculation

\begin{tabular}{|c|c|c|c|}
\hline \multicolumn{2}{|l|}{ DEAD LOAD } & \multicolumn{2}{|c|}{ LIVE LOAD } \\
\hline Flooring & $3 \mathrm{KN} / \mathrm{m}$ & \multirow{4}{*}{\multicolumn{2}{|c|}{$\begin{aligned} \text { Live load } & =\text { live load intensity } \mathrm{X} \text { walkway } \\
& =4 \times 2.4 / 2 \\
& =4.8 \mathrm{KN} / \mathrm{m}\end{aligned}$}} \\
\hline Cross girder & $0.0625 \mathrm{KN} / \mathrm{m}$ & & \\
\hline Self-weight of truss & $0.1 \mathrm{KN} / \mathrm{m}$ & & \\
\hline Total load & $3.1625 \mathrm{KN} / \mathrm{m}$ & & \\
\hline Factored Load & $4.75 \mathrm{KN} / \mathrm{m}$ & Factored load & $7.2 \mathrm{KN} / \mathrm{m}$ \\
\hline
\end{tabular}

Above Table 1 showing Dead load and Live load calculation of foot-over bridge.

Table 2: Influence Line Diagram [1,8]

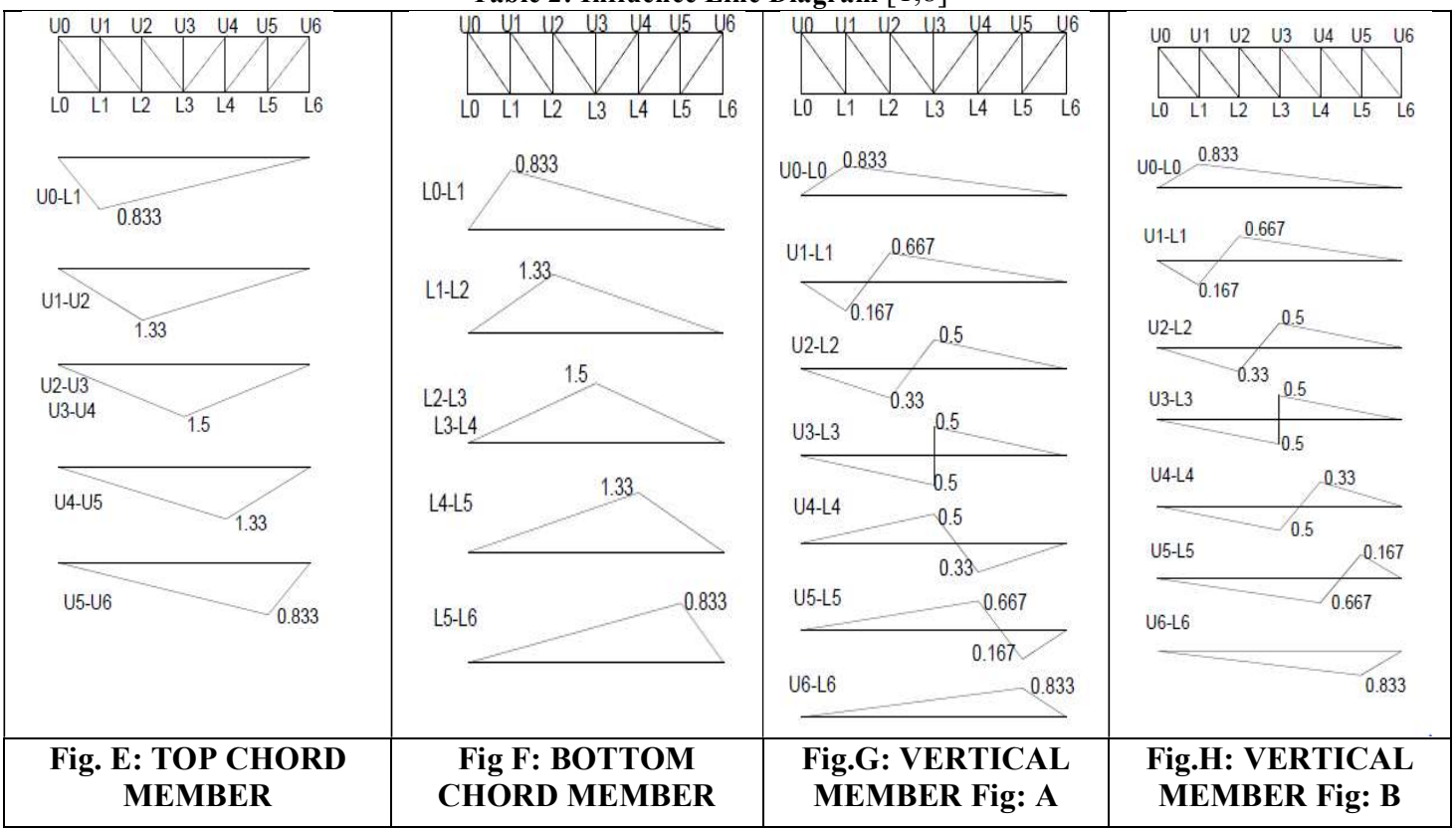

ILD of Top chord (Fig:1) and Bottom Chord (Fig:2) will be same for all the figures A,B,C,D

Table 3: Influence Line Diagram $[4,6]$

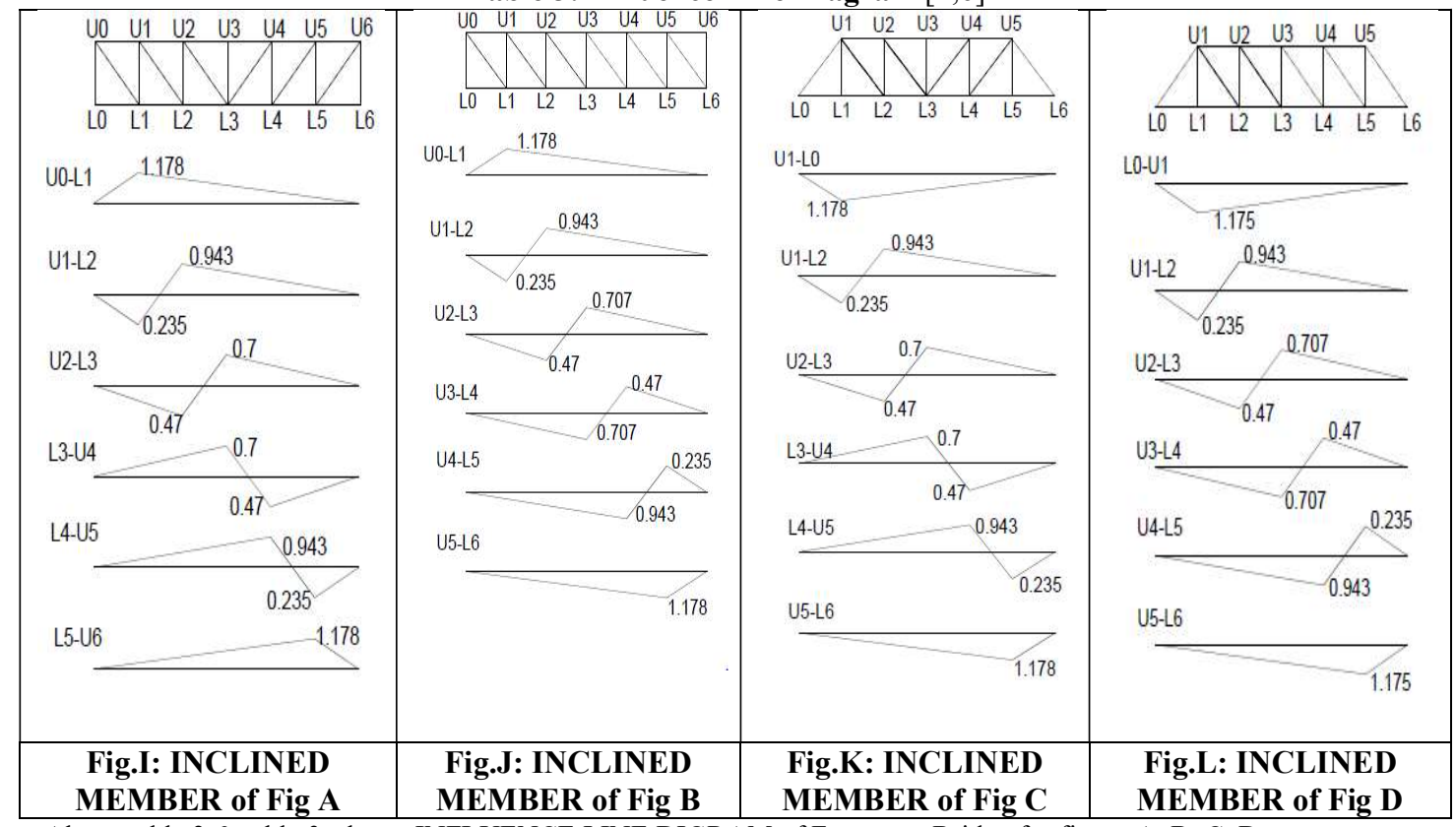

Above table $2 \&$ table 3 , shows INFLUENCE LINE DIGRAM of Foot-over Bridge for figure A, B, C, D. 
Sample Calculations $[1,6]$

Forces in Inclined Member: U1-L2 for figure A, B, C, D

Fdead $=4.75 \times(-0.5 \times 2.4 \times 0.235+0.5 \times 9.6 \times 0.943)=20.16 \mathrm{KN}$

Flive $1=(-0.5 \times 2.4 \times 0.235 \times 7.2)=-2.0304 \mathrm{KN}$

Flive $2=0.5$ X 9.6 X $0.943 \times 7.2=32.59 \mathrm{KN}$

Net Forces $=$ Dead Load + Live Load;

$\mathrm{F} 1=20.106-2.304=\mathbf{1 8 . 0 7} \mathbf{K N}(\mathbf{T}) \& \mathrm{~F} 2=20.106+32.59=\mathbf{5 2 . 7} \mathbf{K N}(\mathbf{T})$

Table 4: Vertical Members

\begin{tabular}{|l|l|l|l|l|l|l|l|l|}
\hline $\begin{array}{l}\text { MEMBE } \\
\text { R }\end{array}$ & \multicolumn{2}{|c|}{ FIGURE A } & \multicolumn{2}{c|}{$\begin{array}{l}\text { FIGURE } \\
\text { B }\end{array}$} & \multicolumn{2}{c|}{ FIGURE C } & \multicolumn{2}{c|}{ FIGURE D } \\
\hline U0 -L0 & $\mathbf{5 9 . 5 ( T )}$ & - & $59.5(\mathrm{~T})$ & - & - & - & - & - \\
\hline U1 -L1 & $12.8(\mathrm{~T})$ & $37.3(\mathrm{~T})$ & $12.81(\mathrm{~T})$ & $37.30(\mathrm{~T})$ & $12.8(\mathrm{~T})$ & $37.30(\mathrm{~T})$ & $12.81(\mathrm{~T})$ & $37.3(\mathrm{~T})$ \\
\hline U2 -L2 & $0.88(\mathrm{C})$ & $17.7(\mathrm{~T})$ & $0.89(\mathrm{C})$ & $17.77(\mathrm{~T})$ & $0.88(\mathrm{C})$ & $17.78(\mathrm{~T})$ & $0.89(\mathrm{C})$ & $17.7(\mathrm{~T})$ \\
\hline U3 -L3 & $10.8(\mathrm{C})$ & $10.8(\mathrm{~T})$ & $10.8(\mathrm{C})$ & $10.8(\mathrm{~T})$ & $10.8(\mathrm{C})$ & $10.8(\mathrm{~T})$ & $10.8(\mathrm{C})$ & $10.8(\mathrm{~T})$ \\
\hline U4 -L4 & $17.7(\mathrm{~T})$ & $0.89(\mathrm{C})$ & $17.78(\mathrm{C})$ & $0.899(\mathrm{~T})$ & $17.7(\mathrm{~T})$ & $0.89(\mathrm{C})$ & $17.7(\mathrm{C})$ & $0.89(\mathrm{~T})$ \\
\hline U5 -L5 & $37.3(\mathrm{~T})$ & $12.1(\mathrm{~T})$ & $37.30(\mathrm{C})$ & $12.81(\mathrm{C})$ & $37.3(\mathrm{~T})$ & $12.81(\mathrm{~T})$ & $37.3(\mathrm{C})$ & $12.8(\mathrm{C}$ \\
\hline U6 -L6 & $59.5(\mathrm{~T})$ & - & $\mathbf{5 9 . 5}(\mathrm{C})$ & - & - & - & - & - \\
\hline
\end{tabular}

Above Table: 4 shows compressive \& tensile forces of Vertical Member for figure A, B, C, D

Table 5: Top Chord Member

\begin{tabular}{|l|l|l|l|l|l|l|l|l|}
\hline MEMBER & \multicolumn{2}{|c|}{ FIGURE A } & \multicolumn{2}{c|}{ FIGURE B } & \multicolumn{2}{c|}{ FIGURE C } & \multicolumn{2}{c|}{ FIGURE D } \\
\hline U0-U1 & $59.5(\mathrm{C})$ & & $59.5(\mathrm{C})$ & & & & & \\
\hline U1-U2 & $95.36(\mathrm{C})$ & & $95.36(\mathrm{C})$ & & $95.36(\mathrm{C})$ & & $95.36(\mathrm{C})$ & \\
\hline U2-U3 & $107.55(\mathrm{C})$ & & $107.55(\mathrm{C})$ & & $107.55(\mathrm{C})$ & & $107.55(\mathrm{C})$ & \\
\hline U3-U4 & $107.55(\mathrm{C})$ & & $107.55(\mathrm{C})$ & & $107.55(\mathrm{C})$ & & $107.55(\mathrm{C})$ & \\
\hline U4-U5 & $95.36(\mathrm{C})$ & & $95.36(\mathrm{C})$ & & $95.36(\mathrm{C})$ & & $95.36(\mathrm{C})$ & \\
\hline U5-U6 & $59.5(\mathrm{C})$ & & $59.5(\mathrm{C})$ & & - & & - & \\
\hline
\end{tabular}

Above Table: 5 shows compressive \& tensile forces of Top Chord Member for figure A, B, C, D

Table 6: Bottom Chord Member

\begin{tabular}{|c|c|c|c|c|c|}
\hline MEMBER & FIGURE A & FIGURE B & FIGURE C & \multicolumn{2}{|c|}{ FIGURE D } \\
\hline L0-L1 & $59.5(\mathrm{~T})$ & $59.5(\mathrm{~T})$ & $59.5(\mathrm{~T})$ & $59.5(\mathrm{~T})$ & \\
\hline L1-L2 & $95.36(\mathrm{~T})$ & $95.36(\mathrm{~T})$ & $95.36(\mathrm{~T})$ & $95.36(\mathrm{~T})$ & \\
\hline L2-L3 & $107.55(\mathrm{~T})$ & $107.55(\mathrm{~T})$ & $107.55(\mathrm{~T})$ & $107.55(\mathrm{~T})$ & \\
\hline L3-L4 & $107.55(\mathrm{~T})$ & $107.55(\mathrm{~T})$ & $107.55(\mathrm{~T})$ & $107.55(\mathrm{~T})$ & \\
\hline L4-L5 & $95.36(\mathrm{~T})$ & $95.36(\mathrm{~T})$ & $95.36(\mathrm{~T})$ & $95.36(\mathrm{~T})$ & \\
\hline L5-L6 & $59.5(\mathrm{~T})$ & $59.5(\mathrm{~T})$ & $59.5(\mathrm{~T})$ & $59.5(\mathrm{~T})$ & \\
\hline
\end{tabular}

Above Table: 6 shows compressive \& tensile forces of Bottom Chord Member for figure A, B, C, D

Table 7: Inclined Members

\begin{tabular}{|l|l|l|l|l|l|l|l|l|}
\hline $\begin{array}{l}\text { MEMB- } \\
\text { ER }\end{array}$ & \multicolumn{2}{|c|}{ FIGURE A } & \multicolumn{2}{c|}{ FIGURE B } & \multicolumn{2}{c|}{ FIGURE C } & \multicolumn{2}{c|}{ FIGURE D } \\
\hline & LEFT & RIGHT & LEFT & RIGHT & LEFT & RIGHT & LEFT & RIGHT \\
\hline U0-L1 & $84.46 \mathrm{~T}$ & 0 & $84.46 \mathrm{~T}$ & 0 & & & & \\
\hline U1-L0 & & & & & $84.46 \mathrm{C}$ & & $84.46 \mathrm{C}$ & \\
\hline U1-L2 & $18.07 \mathrm{~T}$ & $52.7 \mathrm{~T}$ & $18.07 \mathrm{~T}$ & $52.7 \mathrm{~T}$ & $18.07 \mathrm{~T}$ & $52.7 \mathrm{~T}$ & $18.07 \mathrm{~T}$ & $52.7 \mathrm{~T}$ \\
\hline U2-L3 & $1.5 \mathrm{C}$ & $24.75 \mathrm{~T}$ & $1.5 \mathrm{C}$ & $24.75 \mathrm{~T}$ & $1.5 \mathrm{C}$ & $24.75 \mathrm{~T}$ & $1.5 \mathrm{C}$ & $24.75 \mathrm{~T}$ \\
\hline U4-L3 & $24.75 \mathrm{~T}$ & $1.5 \mathrm{C}$ & & & $24.75 \mathrm{~T}$ & $1.5 \mathrm{C}$ & & \\
\hline U3-L4 & & & $24.76 \mathrm{C}$ & $1.5 \mathrm{~T}$ & & & $24.76 \mathrm{C}$ & $1.5 \mathrm{~T}$ \\
\hline U5-L4 & $52.7 \mathrm{~T}$ & $18.07 \mathrm{~T}$ & & & $52.7 \mathrm{~T}$ & $18.07 \mathrm{~T}$ & - & - \\
\hline U4-L5 & & & $52.7 \mathrm{C}$ & $18.07 \mathrm{C}$ & & & $52.7 \mathrm{C}$ & $18.07 \mathrm{C}$ \\
\hline U6-L5 & 0 & $84.46 \mathrm{~T}$ & & & & & & \\
\hline U5-L6 & & & 0 & $84.46 \mathrm{C}$ & 0 & $84.46 \mathrm{C}$ & 0 & $84.46 \mathrm{C}$ \\
\hline
\end{tabular}

Above Table: 7 shows compressive \& tensile forces of Inclined Member for figure A, B, C, D 


\section{Design of Truss Members}

a) Compression Member

Initially member of size $60 \times 60 \times 10$ for Top Chord Member, 70 × 70 × 8 for Inclined Member were taken for design, which eventually failed to sustain the loads, in the similar manner we increased the member sizes to check the safety of the member.

The minimum safe sizes for calculated forces in Top Chord Member \& Inclined Member are: 65 x 65 x 8 \& 75 $\mathrm{x} 75 \times 8$ respectively.

Table 8: Compression Member [2,10]

\begin{tabular}{|c|c|c|c|}
\hline & $\begin{array}{l}\text { TOP CHORD } \\
\text { U0-L1 of Fig. A }\end{array}$ & $\begin{array}{ll}\text { INCLINED } & \text { MEMBER } \\
\text { U1-L0 of Fig. C } & \end{array}$ & REFERENCES \\
\hline ANGLE SIZE & $65 \times 65 \times 8$ & $75 \times 75 \times 8$ & \multirow[t]{2}{*}{ Indian Steel Table } \\
\hline $\begin{array}{l}\text { Properties of } \\
\text { Angle }\end{array}$ & $\mathrm{Ag}=976 \mathrm{~mm}^{\wedge} 2, \mathrm{rmin}=11.5 \mathrm{~mm}$ & $\begin{array}{l}\mathrm{Ag}=1138 \mathrm{~mm}^{\wedge} 2 \\
\mathrm{rmin}=14.5 \mathrm{~mm}\end{array}$ & \\
\hline Effective Lentgth & $0.85 \times 2000=1700 \mathrm{~mm}$ & $0.85 \times 2000=1700 \mathrm{~mm}$ & $\begin{array}{l}\text { Clause.7.2.2, Page } \\
: 35, \text { IS } 800-2007\end{array}$ \\
\hline Slenderness Ratio & $\lambda=\operatorname{lmin} / \mathrm{rmin}=136$ & $\lambda=1 \mathrm{~min} / \mathrm{rmin}=117.25$ & $\begin{array}{l}\text { Clause.7.1.2.1, Page } \\
: 34, \text { IS } 800-2007\end{array}$ \\
\hline $\begin{array}{l}\text { Design } \\
\text { Compressive } \\
\text { Stress }\end{array}$ & $\mathrm{Fcd}=69.44 \mathrm{~N} / \mathrm{mm}^{\wedge} 2$ & $\mathrm{Fcd}=90.708 \mathrm{~N} / \mathrm{mm}^{\wedge} 2$ & $\begin{array}{l}\text { Table:9C, Page :42, } \\
\text { IS } 800-2007\end{array}$ \\
\hline $\begin{array}{l}\text { Load Carrying } \\
\text { Capacity }\end{array}$ & $\begin{array}{l}\mathrm{Pu}=69.44 \times 977=67.84 \mathrm{KN}> \\
59.5 \mathrm{KN}\end{array}$ & $\begin{array}{l}\mathrm{Pu}=90.708 \times 1138=103.2 \mathrm{KN} \\
>84.46 \mathrm{KN}\end{array}$ & \\
\hline
\end{tabular}

Above Table: 8 shows calculations for design of compression member i.e. U0-L1 \& U1-L0

\section{B) Tension Member}

On construction site the minimum size of angle used is 50x50x6mm, hence considering this section and checking for the maximum tensile load. Here this section is safe in sustaining the maximum tensile force. Therefore, all members with tensile forces lesser than the Maximum tensile force $(84.46 \mathrm{KN})$ is designed for minimum size of $50 \times 50 \times 6 \mathrm{~mm}$

Table 9: Tension Member [2,9,10]

\begin{tabular}{|c|c|c|}
\hline & TENSION MEMBER & REFERENCES \\
\hline Angle size & $50 \times 50 \times 6 \mathrm{~mm}$ (minimum size) & \multirow{2}{*}{ Indian Steel Table } \\
\hline Gross Area & $568 \mathrm{~mm}^{\wedge} 2$ & \\
\hline $\begin{array}{l}\text { Design Strength due to Yielding of gross section } \\
\quad T_{d g}=A_{g} f_{y} / \gamma_{m o}\end{array}$ & $129.1 \mathrm{KN}>84.46 \mathrm{KN}$ & $\begin{array}{l}\text { Clause.6.2, } \\
\text { page:32 } \\
\text { IS } 800-2007\end{array}$ \\
\hline $\begin{array}{l}\text { Design Strength due to Rupture of critical section } \\
\begin{aligned} \mathrm{T}_{\mathrm{dn}}=0.9 \mathrm{~A}_{\mathrm{nc}} \mathrm{f}_{\mathrm{u}} / \gamma_{\mathrm{m} 1}+\beta \mathrm{A}_{\mathrm{go}} \mathrm{f}_{\mathrm{y}} / \gamma_{\mathrm{mo}} \\
\beta=1.4-0.076(\mathrm{w} / \mathrm{t})\left(\mathrm{f}_{\mathrm{y}} / \mathrm{f}_{\mathrm{u}}\right)\left(\mathrm{b}_{\mathrm{s}} / \mathrm{L}_{\mathrm{c}}\right) \leq\left(\mathrm{f}_{\mathrm{u}} \gamma_{\mathrm{mo}} / \mathrm{f}_{\mathrm{y}} \gamma_{\mathrm{m} 1}\right) \\
\geq(0.7)\end{aligned}\end{array}$ & $\begin{array}{l}\mathrm{Anc}=162 \mathrm{~mm}^{\wedge} 2, \\
\mathrm{Ago}=282 \mathrm{~mm}^{\wedge} 2, \\
\beta=1.114, \\
\mathrm{Tdn}=119.22>84.46 \mathrm{KN}\end{array}$ & $\begin{array}{l}\text { Clause.6.3.3, } \\
\text { page:33 } \\
\text { IS } 800-2007\end{array}$ \\
\hline $\begin{array}{l}\text { Design Strength due to block shear } \\
\mathrm{T}_{\mathrm{db}}=\left(0.9 \mathrm{~A}_{\mathrm{vn}} \mathrm{f}_{\mathrm{u}} /\left(\sqrt{3} \gamma_{\mathrm{m} 1}\right)+\mathrm{A}_{\mathrm{tg}} \mathrm{f}_{\mathrm{y}} / \gamma_{\mathrm{m} 0}\right) \\
\text { or } \\
\mathrm{T}_{\mathrm{db}}=\left(\mathrm{A}_{\mathrm{vg}} \mathrm{f}_{\mathrm{y}} /\left(\sqrt{3} \gamma_{\mathrm{m} 0}\right)+0.9 \mathrm{~A}_{\mathrm{tn}} \mathrm{f}_{\mathrm{u}} / \gamma_{\mathrm{m} 1}\right)\end{array}$ & $\begin{array}{l}\mathrm{Avn}=480 \mathrm{~mm}^{\wedge} 2, \text { Atg }=180 \mathrm{~mm}^{\wedge} 2 \\
T d b=122.7>84.46 \mathrm{KN} \\
\text { Avg }=780 \mathrm{~mm}^{\wedge} 2, \text { Atn }=120 \mathrm{~mm}^{\wedge} 2 \\
T d b=137.77 \mathrm{KN}>84.46 \mathrm{KN}\end{array}$ & $\begin{array}{l}\text { Clause.6.4.1, } \\
\text { page:33 } \\
\text { IS } 800-2007\end{array}$ \\
\hline
\end{tabular}

Above Table: 9 shows calculations for design of Tension member of size 50x50x6mm

\section{Result \& Discussion}

a. In configurations of figure $\mathrm{A} \& \mathrm{C}$ there are more tensile stresses than the compressive stresses.

b. In configurations of figure B \& D there are more compressive stresses than the tensile stresses.

c. Major Tensile \& Compressive Stresses in Inclined members are $84.46 \mathrm{KN} \& 84.46 \mathrm{KN}$ respectively, for all four configurations. 
d. For the same load in tension $(84.46 \mathrm{KN})$ and comparison $(84.6 \mathrm{KN})$ the size of the member in tension (table 9) is comparatively smaller than that in compression (table 8)

Table 10: Cost Comparison [2,10]

\begin{tabular}{|c|c|c|}
\hline$\underline{1}$ & Inclined member for tension force $84.46 \mathrm{KN}(50 \times 50 \times 6 \mathrm{~mm})$ & \multirow{3}{*}{$\begin{array}{l}\text { Percentage Reduction in Steel } \\
1) \text { for Inclined Member } \\
=(25.173-12.727) / 25.173 \\
=0.4934=49.34 \%\end{array}$} \\
\hline & Standard weight $=4.5 \mathrm{~kg} / \mathrm{m} \quad \underline{\text { Weight }=12.727 \mathrm{Kg}}$ & \\
\hline$\underline{2}$ & $\begin{array}{l}\text { Inclined member for compression force } 84.46 \mathrm{KN} \text { Size of } \\
\text { member }=75 \times 75 \times 8 \mathrm{~mm} \text { Weight }=25.173 \mathrm{Kg}\end{array}$ & \\
\hline$\underline{3}$ & $\begin{array}{l}\text { Vertical member for Tension Force } 59.72 \mathrm{KN}(50 \times 50 \times 6) \\
\text { Standard Weight }=4.5 \mathrm{Kg} / \mathrm{m} \text { Weight }=12.727 \mathrm{Kg}\end{array}$ & \multirow[t]{2}{*}{$\begin{array}{l}\text { 2) for Fig A and Fig C end spans } \\
=(40.5279-25.1730) / 40.5279 \\
=0.37=37 \%\end{array}$} \\
\hline$\underline{4}$ & $\begin{array}{l}\text { Top Chord Member for Compression force } 59.51 \mathrm{KN}(65 \times 65 \\
\mathrm{x} 100) \\
\text { Weight }=18.8 \mathrm{Kg}\end{array}$ & \\
\hline
\end{tabular}

Above Table: 10 shows results of cost variation of different configuration of foot-over Bridge

\section{Conclusion}

1. In foot-over bridge tensile force should always be preferred over compressive force.

2. For two members carrying same forces of tension and compression respectively, steel required for tension member is nearly $50 \%$ that of steel required for compression member.

3. As per Configurations of Figure A \& Figure B, there are more compressive forces in figure B compared to that of in figure A, hence Figure A is Preferred.

4. As per Configurations of Figure C \& Figure D, there are more compressive forces in figure D compared to that of in figure $\mathrm{C}$, hence Figure $\mathrm{C}$ is Preferred.

5. On Comparing, the steel required in Figure $\mathrm{C}$ it is nearly $60 \%$ of steel required in Figure $\mathrm{A}$ for the end spans.

6. On comparing all the configurations, Figure $\mathrm{C}$ is most economical and sustainable.

\section{References}

1. Dr. N. Subramanian, ,Code of Practice on Steel Structures -A Review of IS 800: 2007 ${ }^{\circ}$, Computer Design Consultants, Gaithersburg, 20878, USA.

2. IS 800: 2007 Indian Standard "Code of Practice for General Construction in Steel — Code of Practice" (Third Revision).

3. Kushalkumar Yadav, Prashant D Hiwase and Ramesh V. Meghrajani "Study the behaviour of cold form c section purlins under bending” International Journal of Civil Engineering and Technology (IJCIET), Volume 9, Issue 5, May 2018, Pages 964-968 Publisher IAEME Publication.

4. L.S. Negi "Design of Steel Structures", Tata McGraw-Hill Publishing Company (P) LTD, 2nd edition 2008.

5. Limje Mayur, Solanki Dharmendra, Patel Darshan, Patel Neel, Patel Hiren, Chauhan Dixit "Appraisal and Design of Foot Over Bridge”, International Research Journal of Engineering and Technology (IRJET), Volume: 06 Issue: 04 | Apr 2019.

6. S. K. Duggal, "Limit State Design of Steel Structures” McGraw-Hill Education (India) Private LTD 2nd edition, 2010.

7. S. Rajesh, "Design of A Steel Foot Over Bridge in A Railway Station "International Journal of Civil Engineering and Technology (IJCIET) Volume 8, Issue 8, August 2017, pp. 1533-1548, Article ID: IJCIET_08_08_167.

8. S.S Bhavikatti "Design of steel structure by limit state method as per IS - 800: 2007", I.K. International Publishing House Pvt. Ltd. 2009.

9. Sachithanandam P., Meikandaan T.P., Srividya T., Steel framed multi storey residential building analysis and design, International Journal of Applied Engineering Research, v-9, i-22, pp-5527-5529, 2014.

10. Steel Table by S.Ramamrutham, Dhanpat Rai Publishing Company (P) LTD. - 2010 